\title{
Multiple extramedullary plasmacytomas of the trachea and pharyngeal tissue: a case report and literature review
}

This article was published in the following Dove Medical Press journal:

OncoTargets and Therapy

\author{
Ru-rong Tang' \\ Yan Wang ${ }^{2}$ \\ Chao-nan Liang ${ }^{3}$ \\ Wei $\mathrm{Li}^{3}$ \\ Ling Pei ${ }^{1}$ \\ Jian Kang ${ }^{3}$ \\ Gang $\mathrm{Hou}^{3}$
}

'Department of Anesthesiology,

The First Hospital of China Medical

University, Shenyang, China;

${ }^{2}$ Department of Otolaryngology,

The First Hospital of China Medical

University, Shenyang, China;

${ }^{3}$ Department of Respiratory and

Critical Care Medicine, Institute of Respiratory Disease, The First Hospital of China Medical University, Shenyang, China
Correspondence: Gang Hou

Department of Respiratory and Critical

Care Medicine, Institute of Respiratory

Disease, The First Hospital of China

Medical University, No I 55 Nanjing

North Street, Shenyang I 1000 I, China

Tel +862483282530

Fax +86 2483282002

Email hougangcmu@I63.com

\begin{abstract}
Extramedullary plasmacytoma (EMP) is an infrequent form of plasma cell dyscrasia that presents as a mass of monoclonal plasma cells located in extramedullary soft tissues with no skeletal component. EMP constitutes $\sim 4 \%$ of all plasma cell neoplasms and occurs mostly in the upper respiratory tract. Here, we report a rare case of multiple EMPs involving the trachea, laryngopharynx, and oropharynx, which caused symptoms of airway obstruction as the only clinical manifestation. The patient was diagnosed by histopathology of the tissue from bronchoscopic resection and successfully managed with bronchoscopic intervention to treat lesions in the trachea and radiotherapy combined with surgical resection to treat lesions in the pharynx. There was no recurrence after 14 months of follow-up. Endoscopic intervention plays a key role in the rapid diagnosis and treatment of EMP involving the central airways.

Keywords: tracheal tumors, bronchoscopy, interventional pulmonology, cryotherapy, plasmacytoma
\end{abstract}

\section{Introduction}

Extramedullary plasmacytoma (EMP) is a plasma cell malignancy that presents with a soft tissue mass of monoclonal plasma cells, and diagnosis is based on the exclusion of systemic plasma cell disorders. ${ }^{1}$ EMP constitutes $\sim 4 \%$ of all plasma cell neoplasms, occurring mostly in the upper aerodigestive tract (UAD) but also rarely arising in the soft tissues, gastrointestinal tract, skin, and lymph nodes. ${ }^{2}$ The trachea is a very rare site of plasmacytoma, and no more than 20 cases were reported in the medical literature until $2014 .{ }^{3-5}$ Here, we report a case of multiple EMPs involving the trachea, laryngopharynx, and oropharynx that caused symptoms of airway obstruction as the first clinical manifestation. To the best of our knowledge, this is the first case report of EMP occurring in the trachea and laryngopharynx simultaneously; the same lesion subsequently occurred in the oropharynx within a short period of time. We also reviewed a previous study to clarify the clinical manifestations and treatment strategy for EMP involving the respiratory tract. The patient provided written informed consent to have the case report and any accompanying images published. Institutional approval was obtained from the Institutional Ethical Review Board of the First Hospital of China Medical University to publish the case details.

\section{Case presentation}

A 35-year-old man with a 20 pack-year smoking history was admitted to our hospital due to nonproductive cough and dyspnea for 2 months. There was no history of fever, 

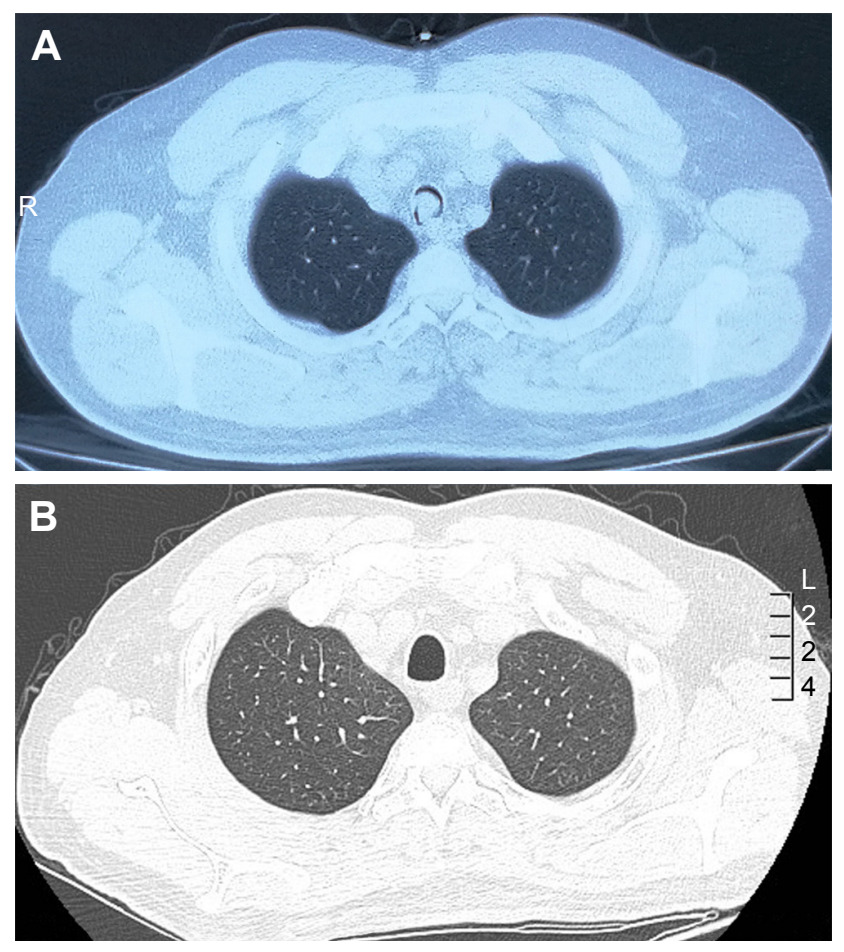

Figure I The manifestations of chest CT.

Notes: (A) Chest CT on admission showed a soft tissue mass in the trachea. (B) Chest CT after radiotherapy showed that the lesion disappeared completely. Abbreviation: CT, computed tomography.

hemoptysis, weight loss, recent change in diet, bone pain, or previous pathological fracture. The patient's vital signs on admission were normal: temperature, $36.5^{\circ} \mathrm{C}$; respiration rate, $16 \mathrm{bpm}$; pulse, $80 \mathrm{bpm}$; and blood pressure, 135/77 $\mathrm{mmHg}$. Blood cell counts, renal function, and liver function were within normal limits, and serum tumor markers were negative.

Chest computed tomography (CT) showed a soft tissue mass in the trachea (Figure 1A). Bronchoscopy revealed two nodular masses with pedicles arising from the posterior wall of the trachea, occluding $\sim 95 \%$ of the tracheal lumen (Figure 2A). We performed rigid bronchoscopy and resected
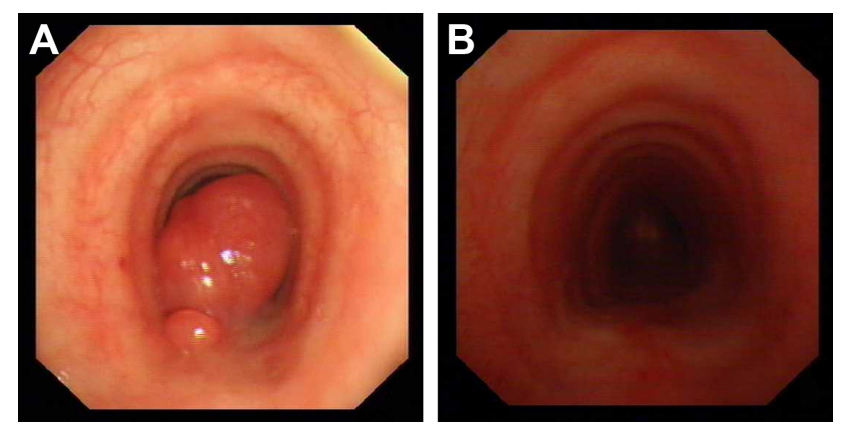

Figure 2 The bronchoscopic manifestations of the lesion.

Notes: (A) Bronchoscopy revealed two nodular masses with pedicles arising from the posterior wall of the trachea, occluding $\sim 95 \%$ of the tracheal lumen. (B) At the end of 14-month follow-up, repeated bronchoscopy revealed complete recovery of the lesion.
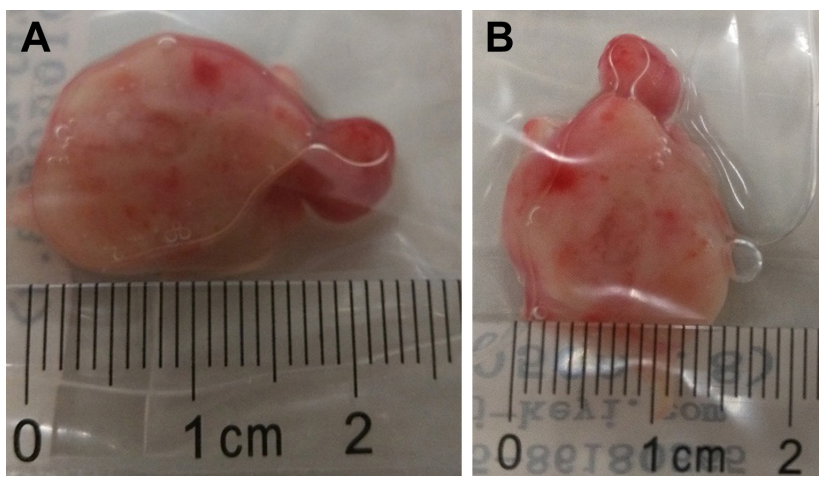

Figure 3 The size of the excised tumor.

Notes: (A) The long axis was $2.3 \mathrm{~cm}$. (B) The short axis was $1.5 \mathrm{~cm}$.

the tumor with an electrocautery snare under general anesthesia. We used an argon plasma coagulator to treat the base of the residual tumor. The excised tumor measured $2.3 \times 1.5 \mathrm{~cm}$ in diameter (Figure 3 ), and the patient's respiratory symptoms were relieved significantly. Pathological examination with immunohistochemical staining diagnosed the mass as plasmacytoma (Figure 4).

Then, a multiple myeloma (MM) workup was performed. The bone marrow aspirate and biopsy showed normal morphology. The blood cell cluster differentiation antigen series and fluorescence in situ hybridization (FISH) detection (MM group set) with bone marrow were normal. The results of urinalysis for the Bence-Jones protein, serum electrophoresis, and serum protein electrophoresis were all negative. For a more comprehensive examination, the patient underwent an 18-fluorodeoxyglucose positron emission tomography (18FDG-PET/CT) imaging scan, which showed localized and increased FDG uptake within the anterior and posterior walls of the laryngopharynx (Figure 5), with no abnormalities in the bone or other tissues. After all these investigations, the diagnosis of MM was excluded, and EMP was eventually confirmed.

Laryngoscopy was done (Figure 6A), and histopathology of the laryngeal biopsies showed that many submucosal masses of plasma cells proliferated. In view of the residual postoperative lesion in the trachea and the suspicious lesions in the laryngopharynx, the patient was managed with radiotherapy at a dose of 48 Gy over 4 weeks (Figure 2B).

At the end of radiotherapy, the patient's repeated CT (Figure 1B) and laryngoscopy demonstrated that the original lesions had disappeared, but two adjacent smooth neoplasms were observed on the back of the soft palate (Figure 6B). MRI showed the tumor located in the mucosa of the left oropharynx, which was $\sim 7 \mathrm{~mm}$ in diameter (Figure 7). After a comprehensive assessment, the patient underwent neoplasm resection via hypothermic plasma 


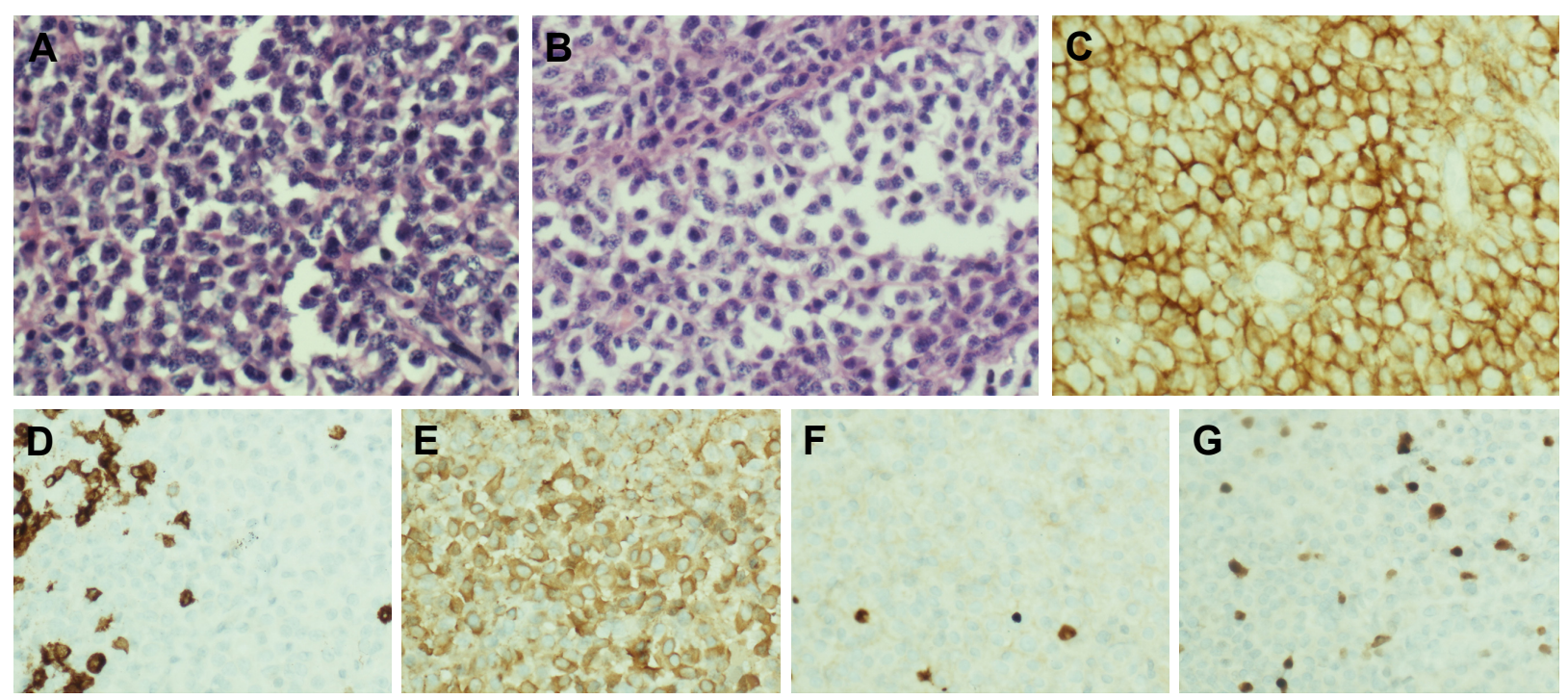

Figure 4 Histopathology of the tumor.

Notes: (A) The plasma cells are relatively uniform and most have eccentrically located nuclei (H\&E, 400X). (B) The nuclear chromatin is dispersed, and a prominent centrally located nucleolus can be found in each nucleus (H\&E, 400x). Immunohistochemical staining showed (C) expression of CD38, (D) absence of CD20, (E) cytoplasmic lambda light chain positivity, and (F) absence of kappa light chain expression. (G) The MIB-I (Ki67) index is $\sim 10 \%$.
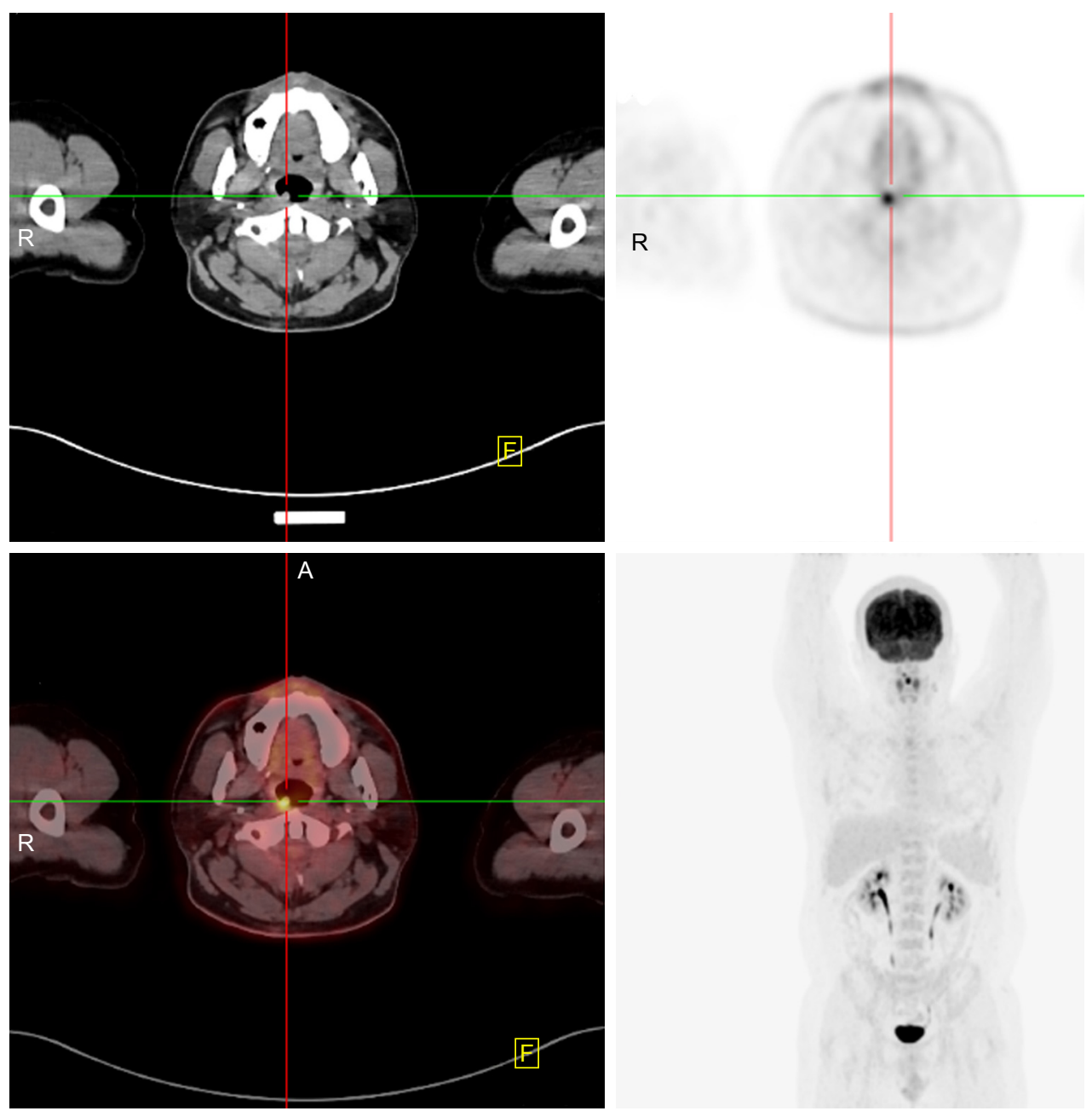

Figure 5 The manifestation of I8FDG-PET/CT.

Note: I8FDG-PET/CT showed localized and increased FDG uptake within the anterior and posterior walls of the laryngopharynx.

Abbreviation: 18FDG-PET/CT, 18-fluorodeoxyglucose positron emission tomography. 

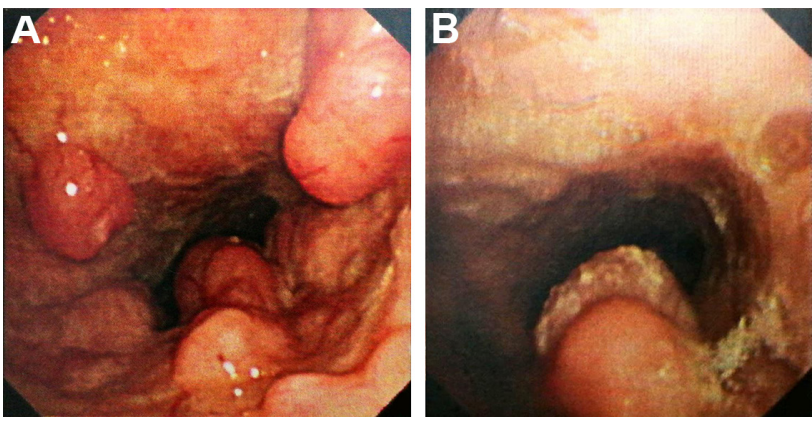

Figure 6 The laryngoscopic manifestations of the tumor.

Notes: (A) Laryngoscopy showed multiple nodules located in the laryngopharynx. (B) Laryngoscopy after radiotherapy showed two adjacent smooth neoplasms on the back of the soft palate.

radiofrequency under general anesthesia. Histopathology and immunohistochemistry of the excised tissue proved that the tumor was plasmacytoma. The patient responded well to the treatment and had no recurrence at the 14-month follow-up.

\section{Discussion}

EMP is a rare tumor that was first reported by Schridde in 1905. ${ }^{6}$ With the assistance of adhesion molecules, pathologic plasmablasts may settle in soft tissue or in an extracellular connective tissue area, which is the origin of EMP. ${ }^{2}$ The etiology of these lesions is not well understood, but viral pathogenesis and chronic irritation have been suggested to be contributing factors. ${ }^{3,7}$ The median age of presentation of EMP is 55 years, with a male/female ratio of $3: 1 .^{6}$ The predominant site of EMP is the head and neck region, and tracheal involvement is rare, constituting only $0.1 \%$ of all malignancies. ${ }^{1,3}$

The most frequent clinical symptoms in UAD are airway obstruction, epistaxis, rhinorrhea, pain, and swelling of the soft tissue. ${ }^{5,8}$ When EMP occurs in the trachea, dyspnea is the most common symptom and becomes more severe
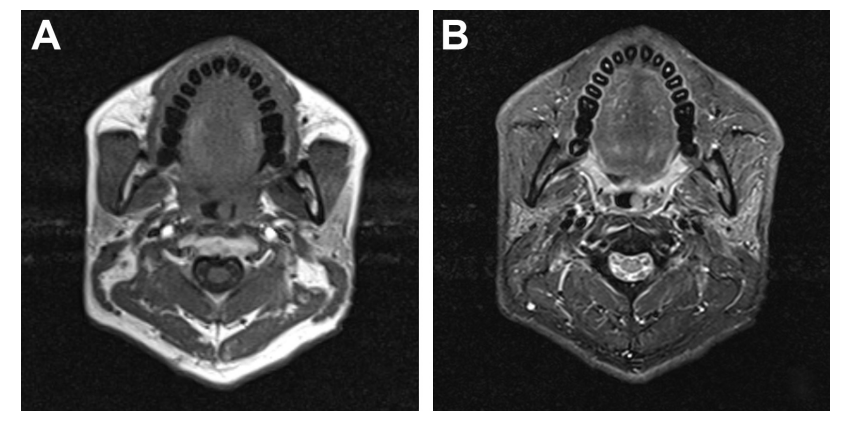

Figure 7 Enhanced MRI of laryngopharynx.

Notes: $(\mathbf{A})$ MRI of the nasopharynx showed a round tumor located in the mucosa of the left oropharynx, $\sim 7 \mathrm{~mm}$ in diameter, that had homogeneous signal intensity similar to the signal intensity of the mucosa on $\mathrm{TI}$-weighted imaging and was enhanced with contrast. (B) On T2-weighted imaging, the signal intensity of the tumor was moderately higher than that of the mucosa. when the tracheal lumen is narrowed by $>75 \% .{ }^{9,10}$ In our case, airway obstruction was the only clinical manifestation before diagnosis, although the laryngopharynx and oropharynx showed swelling of the mucosa, which was found accidentally in the subsequent examination.

$\mathrm{CT}$ and MRI are useful for assessing the local extent and severity of EMP. MRI offers advantages over CT due to its high soft tissue contrast and multiplanar depictions. On MRI, EMPs are typically hypointense on T1-weighted images, enhanced with contrast and hyperintense on T2-weighted images, with short tau inversion recovery sequences. Therefore, MRI is advised as a routine staging procedure during the workup of EMP. ${ }^{11}$ 18FDG-PET/CT examines the whole body in a faster and single study, with a sensitivity of $96 \%$ and a specificity of $77 \%{ }^{12}$ In fact, PET/CT is mandatory for confirming a diagnosis of EMP according to the updated 2017 International Myeloma Working Group guidelines. ${ }^{13}$ In our case, we used CT, PET/CT, and MRI at different times, which provided sufficient radiographic imaging for diagnosis and for defining the local extent of the EMP. But we should be aware of the complementary effects among different imaging techniques. It is accepted that PET/CT scans have a 30\% more chance of picking up lesions over MRI in some situations although the more accepted understanding is that MRI is complementary to PET/CT and is more capable of discovering small soft tissue lesions (especially in this case), which a PET/CT may not be able to pick up.

The diagnosis of EMP depends on histopathology and immunohistochemistry of tissue biopsies. ${ }^{10,14}$ The presence of a homogenous infiltrate of monoclonal plasma cells, which typically express CD138 and/or CD38, is the characteristic feature of plasmacytoma. ${ }^{15}$ When plasmacytoma is diagnosed, subsequent investigations, including a bone marrow assessment, urine/serum protein electrophoresis, serum electrophoresis, blood cell counts, blood calcium, kidney function measurements, and a skeletal survey, should be performed to rule out MM. ${ }^{15}$ The cytogenetic profiles of plasmacytoma should be evaluated with FISH for bone marrow sample, because the $\mathrm{t}(11 ; 14), \mathrm{t}(4 ; 14)$, del(13q), and $1 \mathrm{q}$ gain were associated with the reoccurrence and prognosis of MM with EMP. ${ }^{16} \mathrm{We}$ did not find significant FISH information for bone marrow, but it was a limitation of our case that we did not evaluate cytogenetic profiles of the tracheal plasmacytoma, which may influence the potential treatment decisions.

As mentioned in our case, endoscopic intervention, which is a relatively safe and less invasive technique, can sufficiently and quickly provide high-quality tissue samples for definite diagnosis and also offers an effective method for removing 
the tumor from the lumen, which relieves symptoms significantly and inhibits the progression of the disease.

EMP is highly sensitive to radiotherapy. A total fractionated dose of 40-50 Gy is the common dose for EMP according to the published guidelines. The National Comprehensive Cancer Network recommended a minimum dose of 40 Gy regardless of tumor size, which was similar to the UK Myeloma Forum's guidelines. ${ }^{17,18}$ Guidelines from the International Lymphoma Radiation Oncology Group recommended total dose 40-50 Gy for solitary EMP. But in cases of small, well-defined, or postexcision with positive margins, 40 Gy was acceptable. The dose of radiotherapy for EMP was not in consensus yet. ${ }^{11}$ The optimal dose of radiotherapy should be assessed in future because of the ceiling effect of dose-response relationship. Surgery might make it possible to resect large and well-defined masses but should be followed by radiotherapy..$^{15}$ Our patient showed a better clinical response to bronchoscopic intervention combined with radiotherapy, radiotherapy alone, and surgery alone in the different location and without recurrence during a 14-month follow-up. EMP is usually indolent and localized, with a rate of conversion to MM of $15 \%-20 \%{ }^{19}$ The conversion risk is highest during the first 2 years, and long-term follow-up is needed. ${ }^{20}$

\section{Conclusion}

Multiple EMPs of the trachea and pharynx are extremely rare. Endoscopic intervention plays a key role in the diagnosis and removal of the tumors. The combination of this approach with radiotherapy may provide a better outcome for patients with EMP.

\section{Disclosure}

The authors report no conflicts of interest in this work.

\section{References}

1. Soutar R, Lucraft H, Jackson G, et al. Guidelines on the diagnosis and management of solitary plasmacytoma of bone and solitary extramedullary plasmacytoma. Br J Haematol. 2004;124(6):717-726.

2. Alexiou C, Kau RJ, Dietzfelbinger H, et al. Extramedullary plasmacytoma: tumor occurrence and therapeutic concepts. Cancer. 1999;85(11):2305-2314.

OncoTargets and Therapy

\section{Publish your work in this journal}

OncoTargets and Therapy is an international, peer-reviewed, open access journal focusing on the pathological basis of all cancers, potential targets for therapy and treatment protocols employed to improve the management of cancer patients. The journal also focuses on the impact of management programs and new therapeutic agents and protocols on
3. Sukumaran R, Nair RA, Jacob PM, Koshy SM, Mathew AP. Extramedullary plasmacytoma of the trachea. Head Neck Pathol. 2014;8(2): 220-224.

4. Stevic R, Ercegovac M, Stojšić J, Čolić N. Rare tracheal tumor: solitary plasmacytoma. J Postgrad Med. 2018;64(2):115-118.

5. Rai SP, Kumar R, Bharadwaj R, Panda BN. Plasmacytoma stracheal solitary tracheal plasmacytoma. Indian J Chest Dis Allied Sci. 2003; 45(4):269-272.

6. Soutar R, Lucraft H, Jackson G, et al. Guidelines on the diagnosis and management of solitary plasmacytoma of bone and solitary extramedullary plasmacytoma. Clin Oncol. 2004;16(6):405-413.

7. Lomeo PE, McDonald JE, Finneman J. Extramedullary plasmacytoma of the nasal sinus cavities. Am J Otolaryngol. 2007;28(1): $50-51$.

8. Byrd RP, Roy TM, Bentz W, Mehta JB. Plasmacytoma as a cause of obstructive sleep apnea. Chest. 1996;109(6):1657Jr-1659Jr.

9. Duarte ML, de Brito MC, Traple FA, Prado JL, Scoppetta LC. Plasmacytoma of the trachea: a surprising diagnosis. Radiol Bras. 2017;50(1): 66-67.

10. Garelli M, Righini C, Faure C, Jankowski A, Brambilla C, Ferretti GR. Imaging of a case of extramedullary solitary plasmacytoma of the trachea. Case Rep Radiol. 2011;2011(4):1-4.

11. Tsang RW, Campbell BA, Goda JS, et al. Radiation therapy for solitary plasmacytoma and multiple myeloma: guidelines from the International lymphoma radiation Oncology Group. Int J Radiat Oncol Biol Phys. 2018;101(4):794-808.

12. Gautam A, Sahu KK, Alamgir A, Siddiqi I, Ailawadhi S. Extramedullary solitary plasmacytoma: demonstrating the role of ${ }^{18} \mathrm{~F}-\mathrm{FDG}$ PET imaging. J Clin Diagn Res. 2017;11(4):XD01-XD03.

13. Cavo M, Terpos E, Nanni C, et al. Role of ${ }^{18} \mathrm{~F}-\mathrm{FDG}$ PET/CT in the diagnosis and management of multiple myeloma and other plasma cell disorders: a consensus statement by the International Myeloma Working Group. Lancet Oncol. 2017;18(4):e206-e217.

14. Dulmet E, Verley JM, Levasseur P, Jaubert F, Choudat L. [Solitary plasmacytoma of the trachea. Apropos of a case]. Ann Pathol. 1990;10(4): 275-277. French.

15. Caers J, Paiva B, Zamagni E, et al. Diagnosis, treatment, and response assessment in solitary plasmacytoma: updated recommendations from a European expert panel. J Hematol Oncol. 2018;11(1):10.

16. Shin HJ, Kim K, Lee JJ, et al. The t(11;14)(q13;q32) translocation as a poor prognostic parameter for autologous stem cell transplantation in myeloma patients with extramedullary plasmacytoma. Clin Lymphoma Myeloma Leuk. 2015;15(4):227-235.

17. Kumar SK, Callander NS, Alsina M, et al. Multiple myeloma, version 3.2017, NCCN clinical practice guidelines in oncology. J Natl Compr Canc Netw. 2017;15(2):230-269.

18. Soutar R, Lucraft H, Jackson G, et al. Guidelines on the diagnosis and management of solitary plasmacytoma of bone and solitary extramedullary plasmacytoma. Br J Haematol. 2004;124(6): 717-726.

19. Hazarika P, Balakrishnan R, Singh R, Pujary K, Aziz B. Solitary extramedullary plasmacytoma of the sinonasal region. Indian J Otolaryngol Head Neck Surg. 2011;63(Suppl 1):33-35.

20. Cantone E, Di Lullo AM, Marano L, et al. Strategy for the treatment and follow-up of sinonasal solitary extramedullary plasmacytoma: a case series. J Med Case Rep. 2017;11(1):219.

\section{Dovepress}

patient perspectives such as quality of life, adherence and satisfaction. The manuscript management system is completely online and includes a very quick and fair peer-review system, which is all easy to use. Visit http://www.dovepress.com/testimonials.php to read real quotes from published authors. 